\title{
Virtual Zero Gravity Impact on Internal Gravity Model
}

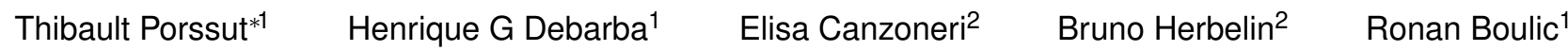 \\ ${ }^{1}$ Immersive Interaction Group, ${ }^{2}$ Center for Neuroprosthetics \\ Ecole Polytechnique Fédéral de Lausanne, Switzerland
}

\begin{abstract}
This project investigates the impact of a virtual zero gravity experience on the human gravity model. In the planned experiment, subjects are immersed with HMD and full body motion capture in a virtual world exhibiting either normal gravity or the apparent absence of gravity (i.e. body and objects floating in space). The study evaluates changes in the subjects' gravity model by observing changes on motor planning of actions dependent on gravity. Our goal is to demonstrate that a virtual reality exposure can induce some modifications to the humans internal gravity model, analogous to those resulting from real exposure (e.g. parabolic flights), even if users remain under normal gravity condition in reality.
\end{abstract}

Index Terms: H.5.1 [Information Interfaces and Presentation]: Multimedia Information Systems-Artificial, augmented, and virtual realities

\section{INTRODUCTION}

Gravity is an important factor for the motion planning performed by the central nervous system (CNS). According to Pozzo et al.[4], CNS explicitly represents gravitational and inertial forces in an internal model used to generate execution plans of limb motions. It is also known that the human gravity model adapts to external conditions, for instance in space or during a parabolic flight $[1,2]$. Although the CNS primarily relies on dedicated inner-ear organs and on proprioception to detect gravity, it has been shown that simple visual feedback of illusory gravity can also influence the motor planning of actions [7]. To investigate if this generalizes to virtual reality feedback, we experimentally induce an immersive virtual zero gravity experience and test if subjects' gravity model adapts accordingly.

\subsection{Related Work}

In a study on human movement prediction, Pozzo et al.[4] asked subjects to extrapolate movement trajectories following biological and non-biological kinematic laws. Results show that the estimation decreases in precision and increases in variability for nonbiological movements. This indicates that motion estimation relies on an internal biological model to map observed actions onto internal representations. This refers to the seminal work of Rizzolatti et al. [6] who famously shown that cortical mechanisms of mirror neurons support abstract representations of movement.

According to Papaxanthis et al. [2], this internal model incorporates both inertial and gravitational forces. The influence of shortterm exposure to zero gravity over these factors was demonstrated in an experiment exposing subjects to parabolic flights (several micro gravity phases of 30s). Under such conditions, the kinematic properties of pointing movements is slightly altered, suggesting that the experience of zero gravity affects the internal gravity model, which in turn influences the motor planning.

*e-mail: thibault.porssut@epfl.ch

2017 IEEE Virtual Reality (VR)

March 18-22, 2017, Los Angeles, CA, USA

978-1-5090-6647-6/17/\$31.00 (C) 2017 IEEE
Interestingly, Sciutti et al. [7] showed that visual indications of gravity also influence gravity model and movement planning. To demonstrate this, the authors asked subjects to perform horizontal arm pointing movements while hiding the real arm, showing instead vertical movements. Results show that subjects adapted the kinematic of the motor plan to include gravitational forces, although this is not physically optimal and only due to an illusory gravity.

\subsection{Contribution}

We developed a simulation, using first-person perspective immersion and avatar embodiment, to study experimentally the influence of an apparent absence of gravity (virtual zero gravity) on the internal gravity model. Following the work on actual zero gravity presented in $[7,4,2]$, we hypothesize that the kinematic of pointing movement might be influenced by virtual zero gravity, thus showing an adaption of the internal gravity model during VR immersion.

\section{Virtual EnVironment Design and Implementation}

We immerse subjects with a full body motion tracking system and Head Mounted Displays (HMD). We combine these devices with a first person view on the avatar, thus increasing the feeling of body ownership and the effectiveness of our VR experiment [3].

\subsection{Development Tools}

The application is implemented in Unity 3D (5.4.1f1). Images are rendered in the screens of the Oculus Rift (Consumer version 1, $1080 \times 1200$ screen resolution per eye, refresh rate of $90 \mathrm{~Hz}, 110^{\circ}$ field of view). We use Bose QuietComfort 15 acoustic noise cancelling headphones to stream an acoustic white noise and avoid any auditory disturbances.

Full body motion capture is done with PhaseSpace ImpulseX2 motion capture system. Subjects wear a suit equipped with LEDs placed on body segments (see the Figure 1). The tracking area is $2.5 \mathrm{~m}$ by $2.5 \mathrm{~m}$, providing users with a large space to explore the zero gravity environment. A calibration phase is required to reconstruct the posture of the avatar. For this purpose, we use our custom calibration tool and an analytic inverse kinematics solver (Figure1).
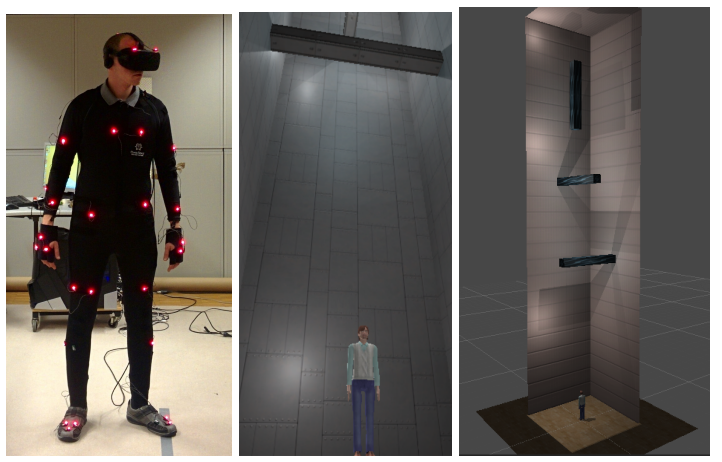

Figure 1: Subject wearing motion capture suit and the HMD (left) and the virtual environment (middle \& right). 


\subsection{Zero Gravity Application}

The virtual environment consists in a rectangular room (Figure1). Two types of randomly generated spheres populate the environment; the target spheres (red) give one point and disappear once touched, and the normal spheres (grey) give no score and bounce once touched. Subjects are instructed to hit as many red spheres as possible during a limited time. All these spheres are affected by gravity settings (and also float and bounce between them and against the wall). Three obstacles bars are placed in the higher part of the room in order to make the avatar and the spheres bounce in the air. These movements give visual cues for providing a convincing illusion of the absence of gravity.

In order to avoid remaining stuck in the air, subjects can trigger a brief force in the upward direction whenever they perform a jump attempt (a sort of "jet backpack" which can be triggered at will).

\section{Experiment Protocol}

In order to study the influence of the visual experience of zero gravity on subjects, we reproduce in the immersive settings the measures described in $[7,4,2]$. Subjects are exposed to a virtual zero gravity environment in brief repeated blocks, for a duration comparable with [2]. In each block, subjects control the avatar to touch the target spheres while floating in the virtual scene.

\subsection{Measurements}

Motor Measurement: Subjects perform vertical and horizontal pointing movements. We measure the velocity profile of these pointing movements. Under normal conditions, horizontal pointing movements show symmetric velocity profiles, while vertical movements show asymmetric profiles. If virtual zero gravity experience affects participants' gravity model, the vertical pointing movements will have a more symmetric velocity profile.

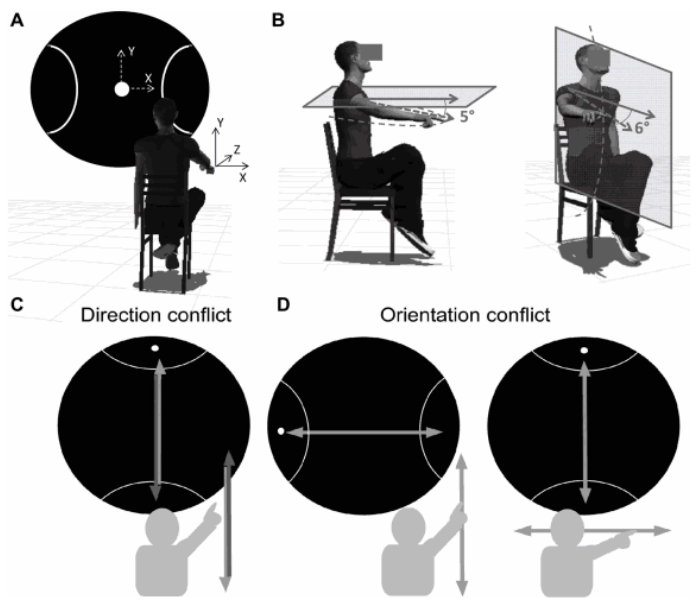

Figure 2: Pointing movement with rotated visual feedback. A and C: visual feedback rotated 180 degrees, causing a direction conflict. B and D: visual feedback rotated 90 degrees, causing an orientation conflict. From [7], with permission of the authors.

Visuo-motor Measurement: Subjects perform vertical and horizontal pointing movements, while seeing a dot moving on a 90 degrees rotated trajectory, reproducing the actual pointing movement (see figure2). After [7], the velocity profile of the horizontal pointing movement will be asymmetric as the visual feedback is vertical (illusion of gravity). Following a virtual zero gravity experience, the velocity profile profile may be less asymmetric (illusion of absence of gravity).

Visual Measurement: The subjects see only a part of biological or non-biological vertical motion trajectories (40\% is occluded) and have to estimate the endpoint of these trajectories. In [4], overestimation was found for both directions, non-biological movements being less precise and variable than biological ones. If the gravity model is altered by virtual zero gravity, the direction of gravity will not match the vertical and the effect will be reduced.

\subsection{Protocols}

The experiment will be performed with 20 participants. The study has been approved by the local Ethical committee. Subjects will give their informed consent before starting the experiment. After a brief preparation phase, subjects will either start with normal gravity condition (control), or zero gravity condition (randomly assigned). They will fill a presence and motion sickness survey at the end of the experiment.

Preparation: The subject wear the suit and the HMD. The experimenter calibrates the motion capture.

Normal Gravity Simulation: Subjects experience the virtual environment with earth gravity physically simulated and have to get as much score as possible within 4 minutes time. We stop the simulation and show a specific measuring interface to the subject and we give the instruction. Subject performs the three measurements.

Zero Gravity Simulation: Subjects experience the virtual environment with zero gravity configuration and have to get as much points as possible within 25 seconds time. This block is repeated 8 times. Each block is followed by the same measurement procedure as for the normal gravity simulation.

\section{Conclusion}

Pilot experiments allowed us to validate the protocol and the implementation of the measurement tasks. We are currently in the process of gathering data on the 20 subjects for the experiments. We expect to obtain convincing evidence that human gravity model can be altered by the immersion in a virtual zero gravity simulation. The results for the three hypothesis corresponding to the different types of measurements will provide indication on the mechanisms influencing the internal model of gravity. In particular, we hypothesize that the influence of the verticality of a visual feedback (visuomotor and visual measurements) will be reduced after having lost reference to the (visual) vertical in zero gravity, as compared to the normal gravity condition. Overall, showing that an immersive exposure in virtual reality can alter low level mechanisms such as motor planning would be paving the way for the implementation of simple and behavioral measurements of immersion.

\section{REFERENCES}

[1] J. McIntyre, M. Zago, a. Berthoz, and F. Lacquaniti. Does the brain model Newton's laws? Nature neuroscience, 4(7):693-694, 2001.

[2] C. Papaxanthis, T. Pozzo, and J. McIntyre. Kinematic and dynamic processes for the control of pointing movements in humans revealed by short-term exposure to microgravity. Neuroscience, 135(2):371-383, 2005.

[3] V. I. Petkova, M. Khoshnevis, and H. H. Ehrsson. The perspective matters! Multisensory integration in egocentric reference frames determines full-body ownership. Frontiers in Psychology, 2(MAR):1-7, 2011.

[4] T. Pozzo, C. Papaxanthis, J. L. Petit, N. Schweighofer, and N. Stucchi. Kinematic features of movement tunes perception and action coupling. Behavioural Brain Research, 169(1):75-82, 2006.

[5] G. Rizzolatti, L. Fogassi, and V. Gallese. Neurophysiological mechanisms underlying the understanding and imitation of action. Nature Reviews Neuroscience, 2(September):661-70, 2001.

[6] G. Rizzolatti, G. Luppino, and M. Matelli. The organization of the cortical motor system: New concepts. Electroencephalography and Clinical Neurophysiology, 106(4):283-296, 1998.

[7] A. Sciutti, L. Demougeot, B. Berret, S. Toma, G. Sandini, C. Papaxanthis, and T. Pozzo. Visual gravity influences arm movement planning. Journal of neurophysiology, 107(12):3433-45, jun 2012. 